\title{
CORRECTIONS
}

\section{Absolute and relative risks}

The author of this Endgames Statistical Question, Philip Sedgwick, would like to correct an error in the Answers section (BMJ 2012;345:e5613, doi:10.1136/bmj.e5613). In the fourth paragraph of this section, the penultimate sentence should have stated that "the number needed to treat is derived as the reciprocal of the absolute risk reduction; in the example above it is equal to $1 \div 0.044=22.73$ [not " $1 \div 0.44=22.73$ "]."

Cite this as: BMJ 2012;345:e6252

๑ BMJ Publishing Group Ltd 2012 